\title{
The IPoP method to measure Cepheid distances
}

\author{
J. Breitfelder ${ }^{1,2}$, A. Mérand ${ }^{1}$, P. Kervella ${ }^{2}$, and A. Gallenne ${ }^{3}$ \\ ${ }^{1}$ European Southern Observatory, Alonso de Córdova 3107, Casilla 19001, Santiago 19, Chile \\ email: jbreitfe@eso.org \\ ${ }^{2}$ LESIA, Observatoire de Paris, CNRS UMR 8109, UPMC, Université Paris Diderot, 5 place \\ Jules Janssen, 92195 Meudon, France \\ ${ }^{3}$ Universidad de Concepción, Departamento de Astronomía, Casilla 160-C, Concepción, Chile
}

\begin{abstract}
Cepheids are one of the most famous standard candles used to calibrate the Galactic distance scale. However, it is fundamental to develop and test independent tools to measure their distances, in order to reach a better calibration of their period-luminosity (P-L) relationship. We present here the first results obtained with the Integrated Parallax of Pulsation (IPoP) method, an extension of the classical Baade-Wesselink method that derives the distance by making a global modelisation of all the available data. With this method we aim to reach a $2 \%$ accuracy on distance measurements.

Cepheid masses are also an essential key for our comprehension of those objects. We briefly present an original approach to derive observational constraint on Cepheid masses. Unfortunately, it does not lead to promising results.
\end{abstract}

Keywords. gravitation, stars: fundamental parameters, stars: variables: Cepheids

\section{General presentation of the IPoP method}

Cepheids are a fundamental element of the extra-galactic distance ladder, but the calibration of their P-L relationship still has to be improved by measuring independent distances. We present here a strong computing tool (IPoP) that we developed to measure accurate distances of single Galactic Cepheids. This code is based on the classical Baade-Wesselink method but can integrate all the available observables in the modelisation (e.g. magnitudes and colours in all bands, interferometric angular diameters, radial velocities, effective temperatures and spectra), so we can get a good statistical accuracy. The redundancy of part of the data (e.g. photometry and interferometry to estimate the angular diameter) also results in a good robustness of the fitting process. The modelisation is also based on physical models (e.g. ATLAS9 atmospheric models) in order to control systematic errors.

Our method requires a large collection of data, that we mostly gather from online archives. We are also carrying out a large program of Cepheid observations in both hemispheres, with the VLTI and the CHARA interferometers.

\section{Applications}

\subsection{Distance of $\delta$ Cep}

The result that we obtained for $\delta$ Cep is presented in Fig. 1. In this study we used a p-factor of 1.27 (Mérand et al. 2005). We obtain a distance of $d=314 \mathrm{pc}$ with the precision of $3 \%$, consistent with the HST parallax value (Benedict et al. 2002): $\pi=$ $3.66 \pm 0.15$ milli-arcsec $(d=273 \pm 66 \mathrm{pc})$. 

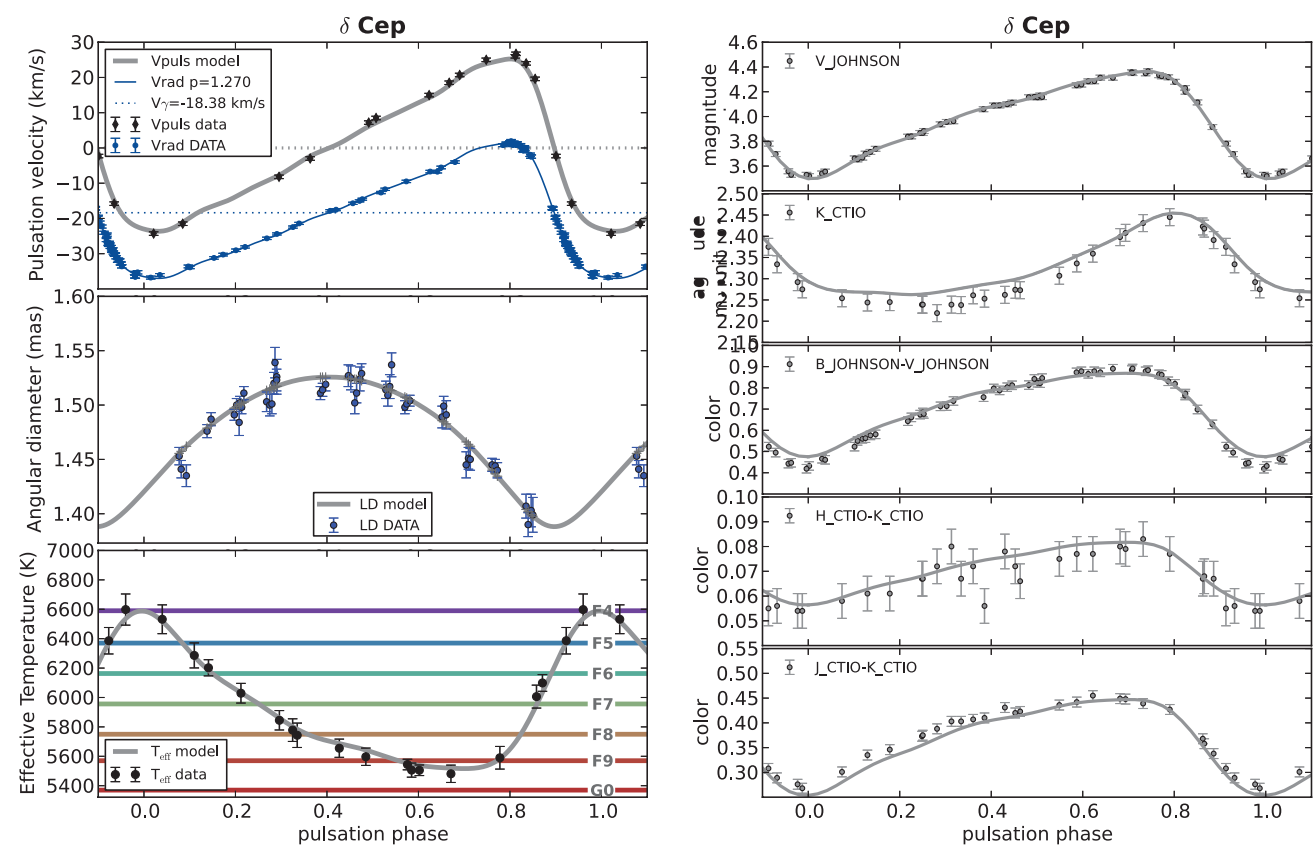

Figure 1. The IPoP method applied to $\delta$ Cep leads to a distance measurement with a $3 \%$ accuracy, $d=314 \pm 10 \mathrm{pc}$.

\subsection{Toward a new constraint on Cepheid masses}

The free fall acceleration, $a_{\mathrm{ff}}$, radius, $R$, and mass, $M$, are linked through: $M=a_{\mathrm{ff}} R^{2} / G$, where $G$ is the gravitational constant. We can rewrite this equation by using our observables and a non-biased distance $d^{\prime}=d / p$, where $p$ is the p-factor, to get a minimum mass, $M_{\min }$ :

$$
M_{\text {min }}=\frac{\theta^{2} d^{\prime 2}}{4 G}\left|\frac{d v_{\mathrm{rad}}}{d t}\right|_{\mathrm{ff}, \max } p^{3},
$$

where $\theta$ is the angular diameter and $\left|d v_{\mathrm{rad}} / d t\right|_{\mathrm{ff}, \max }$, the maximum acceleration in the phase of a contraction of a star.

Considering that other forces can oppose gravitation (e.g. radiation pressure or other pressure forces), this mass can only be considered as an inferior limit of the actual Cepheid mass. We implemented this equation in the IPoP code and found minimum masses much below the masses derived from models (Bono et al. 2011), so not really constraining. During the contraction of the star, the inward acceleration of the atmosphere amounts to about $25 \%$ of the free-fall acceleration, which means that the forces opposing gravity are not negligible. This original method was used by Lacour et al. (2009) to estimate the mass of a Mira star. Assuming a free fall acceleration, they fitted the diameter curve with a parabola to derive the acceleration, and then the corresponding gravitational mass.

\section{References}

Benedict, G. F., McArthur, B. E., Fredrick, L. W., et al. 2002, AJ, 124, 1695

Bono, G., Gieren, W. P., Marconi, M., \& Fouqué, P., Caputo F. 2011, ApJ, 563, 319

Lacour, S., Thiébaut, E., \& Perrin, G. 2009, ApJ, 707, 632

Mérand, A., Kervella, P., Coudé du Foresto, V., et al. 2005, A\&A, 438, L9 